\title{
Development of a three-dimensional dynamic biped walking via the oscillation of telescopic knee joint and its gait analysis
}

\author{
T. Kinugasa ${ }^{1^{*}}$, K. Ando ${ }^{1}$, S. Fujimoto ${ }^{2}$, K. Yoshida ${ }^{1}$ and M. Iribe ${ }^{3}$ \\ ${ }^{1}$ Department of Mechanical Systems Engineering, \\ Okayama University of Science, \\ ${ }^{2}$ Department of Intelligent Mechanical Engineering, \\ Okayama University of Science, \\ 1-1 Ridai-cho, Kita-ku, Okayama, Japan \\ *Email: kinugasa@mech.ous.ac.jp; \\ Phone and Fax: +81-86-256-9531 \\ ${ }^{3}$ Department of Electro-Mechanical Engineering, \\ Osaka Electro-Communication University
}

\begin{abstract}
The purpose of this study is to extend the three-dimensional (3-D) passive dynamic biped walker to a 3-D dynamic biped walker, i.e., a walker that can walk on a horizontal surface based on a passive dynamic walking. A new prototype of 3-D biped walker called RW04, which has telescopic knee joints, was developed and its ability for walking was validated through some experiments. A sinusoidal oscillation, which is regarded as a central pattern generator with no sensory feedback, was provided to the knee joints to achieve the biped walking. The results showed that the biped gait of RW04 was possible only via a sinusoidal oscillation of the knee joint. Moreover, the 3-D dynamic walking gait via frequency response and zero moment point (ZMP) trajectory was also analyzed. The biped locomotion had a resonance, i.e., the frequency matched the natural frequency of the locomotion in the gain property. An " 8 " shaped ZMP trajectory was observed, which was found to be similar to that of the human gait. However, the simple sinusoidal oscillation had limitations such as stride reduction or discontinuation by phase difference. Therefore, in future work, more adaptable control strategy such as a sensory feedback using ZMP should be provided.
\end{abstract}

Keywords: Passive dynamic walking; flat feet; frequency analysis; ZMP.

\section{INTRODUCTION}

In the last two decades, passive dynamic walking (PDW) [1], which was implemented in some walking toys $[2,3]$, has attracted considerable attention of researchers. PDW is a physical phenomenon, and the assumption that biped walking is based on PDW is now widely accepted. A PDW biped changes its gait to adapt to changes in its body configuration and environment, and its efficiency is extremely high. Generally, it is difficult to realize PDW because of the lack of robustness against disturbances. Therefore, biped walkers capable of PDW must be designed carefully, especially for threedimensional (3-D) walking [4-7]. Once a passive dynamic biped walker is realized, it can be expected to show good performance in active biped walking, since its configuration is suitable for biped walking [8-14]. The understanding of our previous PDW bipeds encouraged the authors to extend the study to an active biped walker. Previous studies 
that employed this strategy had successfully achieved 3-D active dynamic biped walking using pneumatic artificial muscles [9-13]. The biped [9-11] was simple, and the objective was to develop an efficient biped walking. The developed bipeds [12,13] possessed many degrees of freedom, and could achieve various movements such as jumping and biped locomotion via the dexterous use of biarticular muscles. However, it is difficult to determine which property of the PDW is essential for an active dynamic biped walking, because of its complexity.

Most of the previous studies on PDW had adopted a spherical sole or circular arc to enable their bipeds to walk stably [9-11]. However, the contacting area between the sole and the ground was too small to provide adequate frictional force against the yaw moment. Moreover, if the radius of the arc foot is small, the biped cannot stand still. On the other hand, flat soles provide higher frictional force with respect to the yaw axis, and they easily enable the biped to stand still in a stable condition. Moreover, ankle springs afford stability similar to that afforded by circular soles. Wisse [15, 16] attempted to implement flat sole and used springs instead of circular soles. The bipeds developed by Hosoda $[12,13]$ also had flat feet actuated by artificial muscles. Accordingly, the authors had previously developed some 3-D PDW bipeds using flat soles and ankle springs and achieved a stable gait [17-19]. The key idea used for achieving a stable 3-D PDW with a flat sole was to tune the position of the ballast attached to each leg. The PDW bipeds remained in the same place by stepping forward and backward because of ground irregularity when we gave an initial oscillation in the lateral direction, even on a horizontal surface. It may appear to be confused, but the authors considered this as an attempt by the biped to walk. There is a well-known method for a planar biped locomotion using cyclic oscillation [7, 20-26]. By providing sensory feedback using foot contact to the dynamics of the nonlinear oscillator, it will be possible for the locomotion to adapt to the environment. It is expected that the 3-D PDW can be extended to an active dynamic 3-D locomotion using this simple input. This study aims for the development of a 3-D active dynamic biped walker called "RW04" based on the knowledge of the previous passive dynamic walker RW03, shown in Figure 1(a) [27], and analyze its gait. Firstly, RW04 was designed and developed with a telescopic knee joint that can perform biped locomotion. Next, it attempted to realize biped locomotion using a simple sinusoidal oscillation of the knee joint. Walking was considered as a frequency response; the knee oscillation and the walking stride were the cyclic input and output, respectively, and the frequency response property of walking was analyzed. Finally, the zero moment point (ZMP) was observed when the biped walks, and the analogy between PDW and active walking was discussed.

\section{3-D DYNAMIC BIPED WALKER RW04}

\section{Prototype of RW04}

There is a method via forced oscillation of a joint by which a passive dynamic walking can be extended to the horizontal surface [7, 20-26]. The authors introduced a telescopic knee joint to RW03 to provide sinusoidal oscillation, and attempted to extend 3-D PDW to a 3-D active dynamic biped walking on a horizontal surface. Figure 1(b) shows the developed biped walker called RW04 that inherits the fundamental configuration of RW03. RW04 had a telescopic knee joint that induced forced oscillation of the knee joint to produce biped locomotion. RW04's height varied from 757 to $807 \mathrm{~mm}$ (RW03: 800 $\mathrm{mm}$ ), and its weight was $6.5 \mathrm{~kg}$ (RW03: $4.3 \mathrm{~kg}$ ). The hip and ankle joints and the foot had the same configuration as in RW03; thus, the hip joint was not actuated (a free joint), the 
ankle joint comprised of a ball joint and coil springs, and the sole of the foot was flat. Movement of the hip joint was observed using a potentiometer. Hip angle was defined by the relative angle between the legs, and measured by the potentiometers on both the hip joints; walking angle was defined by the hip angle when both legs touched the ground. The telescopic knee joint consisted of a DC motor (Maxon: RE30) and a ball screw (THK: VLA-CT-35-21-0050) that can generate a force of $306 \mathrm{~N}$ and move at a speed of 102 $\mathrm{mm} / \mathrm{s}$ (nominal). The knee joint was sufficiently strong against RW04's total weight and can achieve a cycle in $1 \mathrm{~s}$ with an amplitude of $25 \mathrm{~mm}$. The displacement of the knee joint was measured by the encoder (Maxon: MR Type L, 1024 pulses /rotation) attached to the DC motor. The natural frequency of the swing leg was $1.24 \pi \mathrm{rad} / \mathrm{s}$.

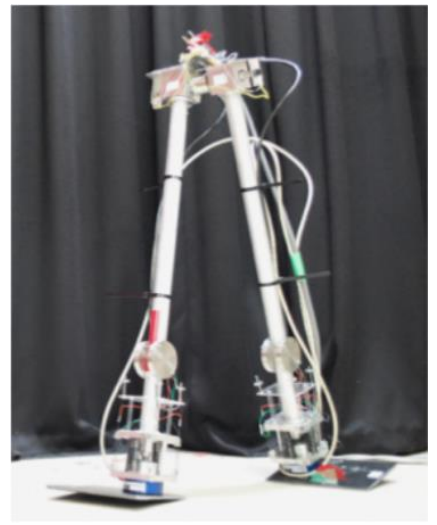

(a)

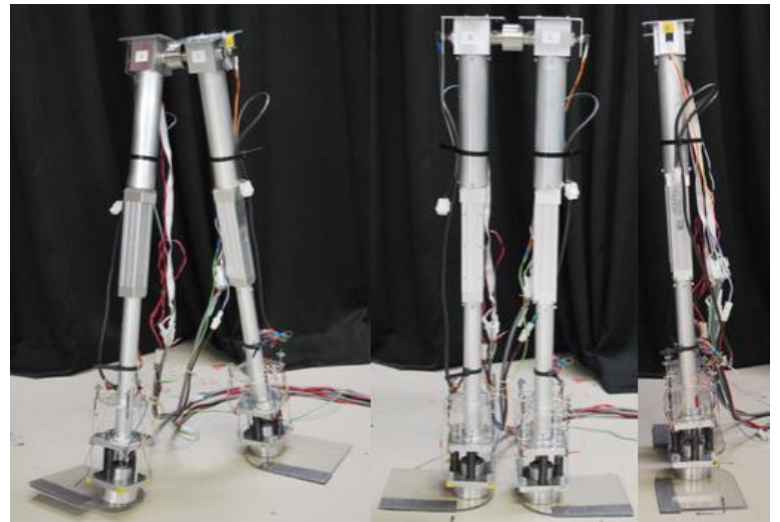

(b)

Figure 1. (a) 3-D PDW: RW03; (b) 3-D dynamic biped walker: RW04.

\section{Strategy for Biped Walking}

The oscillation about the roll axis dominated the 3-D PDW of RW03, and the ballast position determined its amplitude; thus, the height of the leg's center of mass controlled the length of the stride. Therefore, the oscillation of the telescopic knee joint was expected to play a key role in producing the oscillation about the roll axis. Thus, the amplitude and frequency of the telescopic knee joint controlled the amplitude of the swing leg (i.e., stride). To move forward, a spacer was attached to the rear foot spring (the foot spring was located at the front, rear, and outside the ball joint), and RW04 inclined slightly (approximately $3.4^{\circ}$ ) in the forward direction (see right view of Figure 1(b)). The leg swung forward when it lifted off the ground, because of the inclination. Some studies had shown that the variation of a telescopic knee joint can realize a 2-D biped walking on a horizontal surface in a manner similar to the PDW as mentioned above [7, 20-26]. Hence, the same approach had been applied to RW04. To achieve sinusoidal oscillation, the desired trajectory was given by the following sinusoidal functions.

$$
\begin{aligned}
& d_{1}=A \sin \omega t+d_{0} \\
& d_{2}=A \sin (\omega t-\pi)+d_{0}
\end{aligned}
$$

where $d_{1}$ and $d_{2}[\mathrm{~mm}]$ are the displacements of the right and left knees, the origin is the position where the knee is the shortest, $d_{0}[\mathrm{~mm}]$ is the center of oscillation, $A[\mathrm{~mm}]$ is the amplitude, $t[\mathrm{~s}]$ is time, and $\omega[\mathrm{rad} / \mathrm{s}]$ is the frequency. A proportional and derivative (PD) controller is used for positioning and controlling of the knee joint. Eqs. (1) and (2) have 
angle as a linear function of the time; thus, the equations can be rewritten in the following form.

$$
\begin{aligned}
\dot{\zeta} & =\omega, \\
d_{i} & =A \sin \{\zeta-(i-1) \pi\}+d_{0}, i=1,2 .
\end{aligned}
$$

Therefore, the equations can be considered to represent the central pattern generator (CPG) method without a sensory feedback.

\section{EXPERIMENTAL DETAIL}
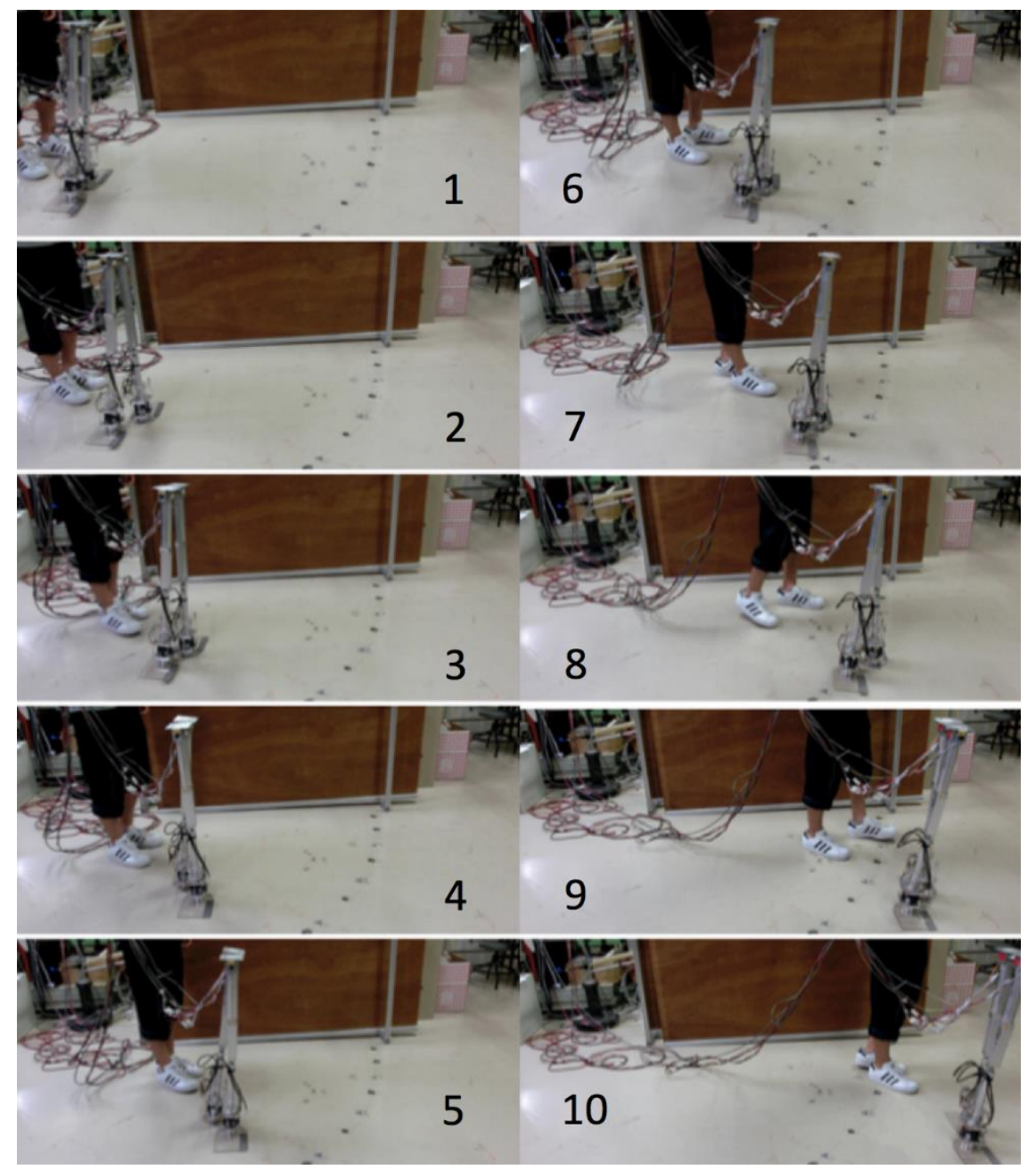

Figure 2. Walking gait of RW04 via sinusoidal knee oscillation.

First, it was confirmed that RW04 could walk via a sinusoidal oscillation of the knee joint. Figure 2 shows sequential screenshots of the walking gait. RW04 walked forward, swaying left and right. The frequency of the knee oscillation was varied from $1.4 \pi \mathrm{rad} / \mathrm{s}$ to $4 \pi \mathrm{rad} / \mathrm{s}$. The amplitude was $5 \mathrm{~mm}$. In the case of $1.6 \pi \mathrm{rad} / \mathrm{s}$, RW04 walked with the maximum walking angle. Figure 3 shows the time response of the hip angle (blue line) and displacements of the knee joints (green and red). The average hip angle was at a maximum when the knee frequency was $1.6 \pi \mathrm{rad} / \mathrm{s}$ (see Figure 3(a)). In this case, the biped walked 22 steps, and the average walking angle was $9.3^{\circ}$. The step number was 
limited by the cable length; thus, it could be expected that the walker could have walked more steps if a treadmill or a wireless control system were used. The frequency of the hip angle movement (corresponding to the walking cycle, which was defined by the time duration for two steps) was found to have variations; however, its average value was identical to that of the knee oscillation. The frequency of moment about the roll axis for the previous passive walker RW03 was $5.24 \mathrm{rad} / \mathrm{s}$, which was similar to that of RW04. Therefore, the active walker RW04 inherited the profile of the passive walker RW03. However, the average walking angle was smaller than that of RW03 $\left(14^{\circ}\right)$. It was found that there was a slight variance in the phase difference between the hip angle and the knee displacement. At around $8 \mathrm{~s}$ and $12 \mathrm{~s}$, the peaks of the hip angle and knee displacements were nearly the same, and the amplitude of the hip angle exceeded the average value. Beyond $9 \mathrm{~s}$, the hip movement was delayed by a few hundred milliseconds from both knees, which resulted in a decrease in its magnitude until $11 \mathrm{~s}$. Figure 3(b) shows the time response of the hip angle when the frequency was $4 \pi$. As mentioned below, the biped could not walk when the frequency was changed from $2.6 \pi$ to $3.2 \pi$. However, the biped could sustain walking when the frequency was $4 \pi$. The frequency of the hip angle was $12.6 \mathrm{rad} / \mathrm{s}$, which was identical to the knee frequency. The walking angle was $2.2^{\circ}$.

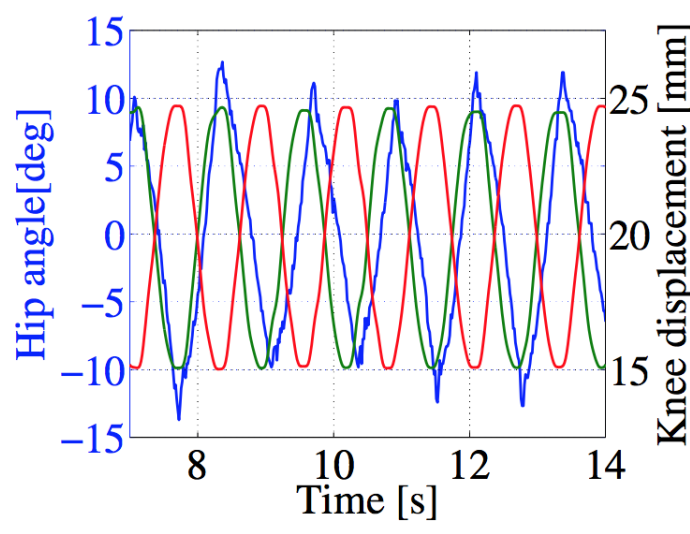

(a)
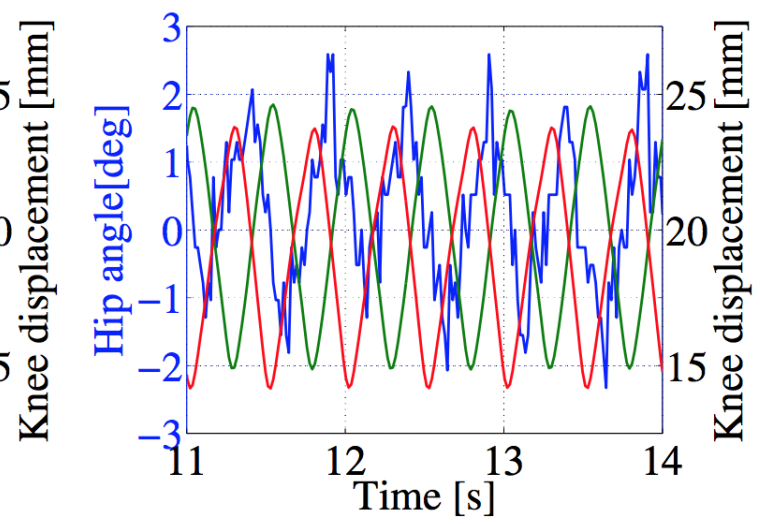

(b)

Figure 3. Time response of hip angle for the frequencies of (a) $1.6 \pi \mathrm{rad} / \mathrm{s}$; (b) $4 \pi \mathrm{rad} / \mathrm{s}$

\section{Frequency Response Analysis}

The frequency response property of the walking gait was analyzed. The frequency of the hip angle was identical to that of the knee displacement, and the amplitude of the hip angle varied with respect to the frequency. Therefore, walking was regarded as a frequency response. The input and output were the knee oscillation and the hip angle, respectively. The amplitude of the knee was fixed at $5 \mathrm{~mm}$. The gain property was expressed by the average walking angle. In the experiment, the trials were repeated five times for each frequency ranging from $1.4 \pi \mathrm{rad} / \mathrm{s}$ to $4 \pi \mathrm{rad} / \mathrm{s}$ with increments of $0.1 \pi$ $\mathrm{rad} / \mathrm{s}$. Figure 4 shows the gain property of the walking gait. The blue circle indicates that the biped can walk more than three times out of the five trials. The red triangle indicates that the biped can walk only less than twice. In the failed case, only successful steps (actually, several steps were available) were used for computing the average walking angle. The black circle shows the case of $4 \pi \mathrm{rad} / \mathrm{s}$. The magenta square indicates the frequency of the passive walker RW03. The frequency varied with respect to the ballast position. A peak was found at approximately $5 \mathrm{rad} / \mathrm{s}$; thus, $5 \mathrm{rad} / \mathrm{s}$ was the natural frequency of RW04. The frequency response property of a 2-D biped walker constrained in the sagittal plane had been shown in the previous studies [24, 25]. However, the peak 
of gain property observed in a numerical simulation could not be found in the experiments for the 2-D biped walker. The results obtained were significant, in the sense that it showed the existence of the peak, i.e., the natural frequency.

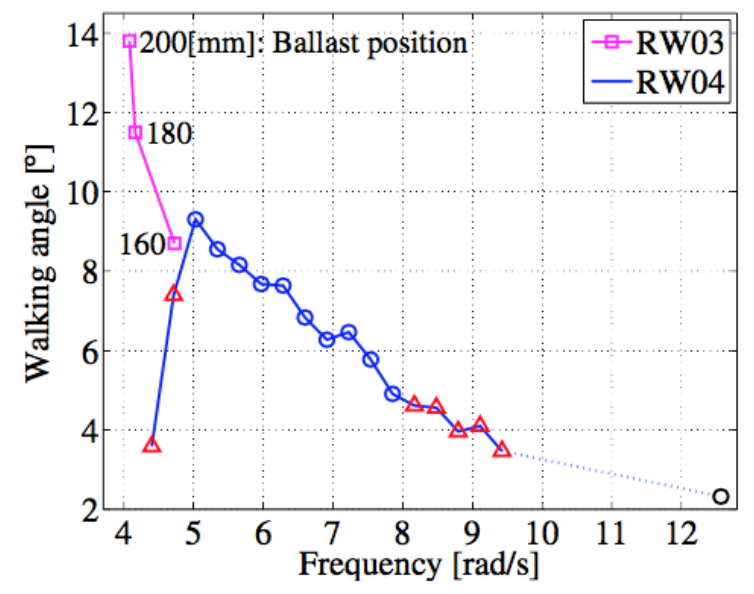

Figure 4. Frequency response property of walking gait.

\section{ZMP Trajectory}

The authors measured the ZMP trajectory when RW04 was walking. The ZMP was calculated using the data measured by a force-torque (FT) sensor (JR3: IFS-67M25A25140-A) attached between the ankle joint and the foot. Figure 5 shows the measured force in the direction of $z$ (vertical) axis (in other words, reaction force), $F_{z}$, and the moments about $x$ - and $y$ - axes (pitch and roll moments) expressed by $M_{x}$ and $M_{y}$, respectively. The reaction force was observed to be lying between two limits; approximately $-3 \mathrm{~N}$ and 61 $\mathrm{N}$. The lower value of $-3 \mathrm{~N}$ indicated the weight of the foot, and the higher value of $61 \mathrm{~N}$ indicated approximately the total weight of RW04, excluding the weight of the foot. Using the data, the equations providing the ZMP are given as

$$
x_{z m p}=\frac{M_{y}}{F_{z}}, y_{z m p}=\frac{M_{x}}{F_{z}}
$$

where $x_{z m p}$ and $y_{z m p}$ are the coordinates of the ZMP position. Figure 6 shows Lissajous patterns of the ZMP for the frequencies of $1.8 \pi, 2.1 \pi$, and $2.4 \pi$. In the figures, the walking direction was towards the left hand side, and blue squares indicate the footprints. The ZMP trajectories drew an " 8 " shape, which was also observed for RW03, and this shape is well known as the profile of the human gait [28]. The trajectories stayed towards the front (right) part of the footprints, because of the forward inclination of RW04 mentioned previously. In the case of $1.8 \pi \mathrm{rad} / \mathrm{s}$ (close to the peak frequency), the trajectory drew the " 8 " shape; however, it tended to fluctuate for each step. In the cases of $2.1 \pi \mathrm{rad} / \mathrm{s}$ and $2.4 \pi \mathrm{rad} / \mathrm{s}$, the trajectory tended to stay in the same area. The area of ZMP decreased when the frequency increased; this was also observed in the frequency response analysis. However, the variation in the direction of $y$ axis was bound from $-0.1 \mathrm{~m}$ to $0.1 \mathrm{~m}$, which was twice smaller than that in the case of RW03 [27]. RW04 had the knee joint that provided foot clearance; thus, RW04 did not require a large lateral oscillation as compared to RW03, which had no knee joint. The result showed that RW04 has a similar profile to the human gait with respect to the " 8 " shaped ZMP trajectory, and that it inherited the traits of RW03. 


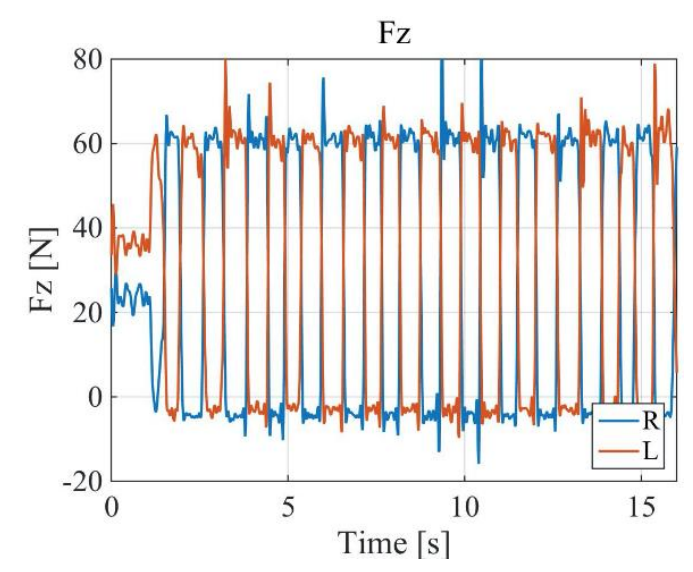

(a)

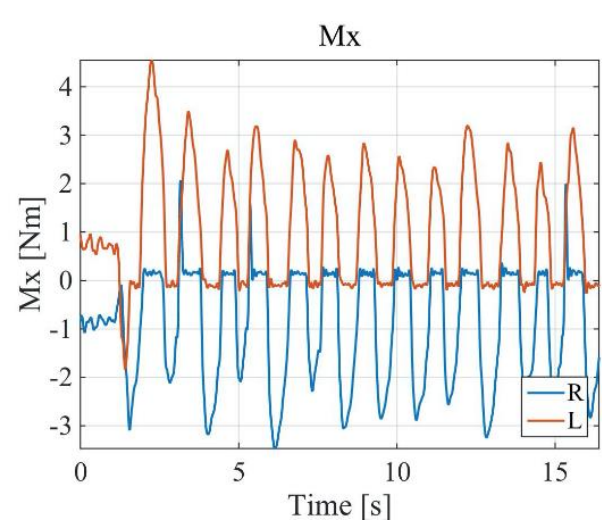

(b)

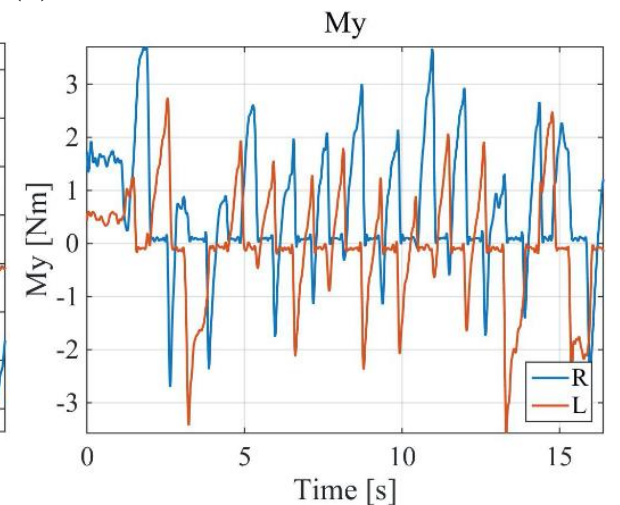

(c)

Figure 5. Force and moments for $1.8 \pi \mathrm{rad} / \mathrm{s}$ measured by FT sensors. (a) Vertical force; (b) moment about $x$-axis; (c) moment about $y$-axis.

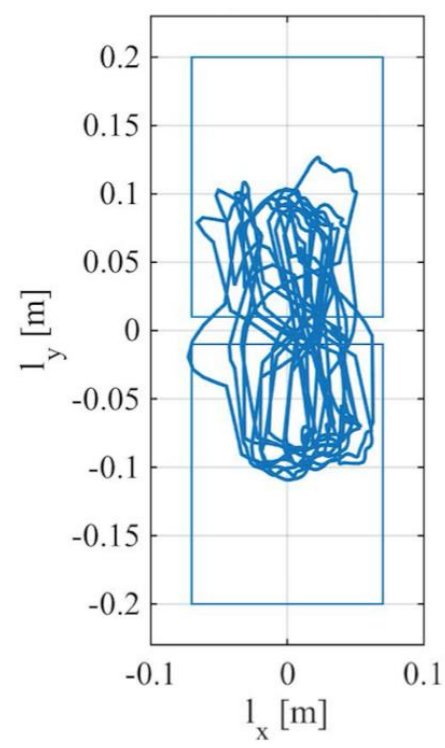

(a)

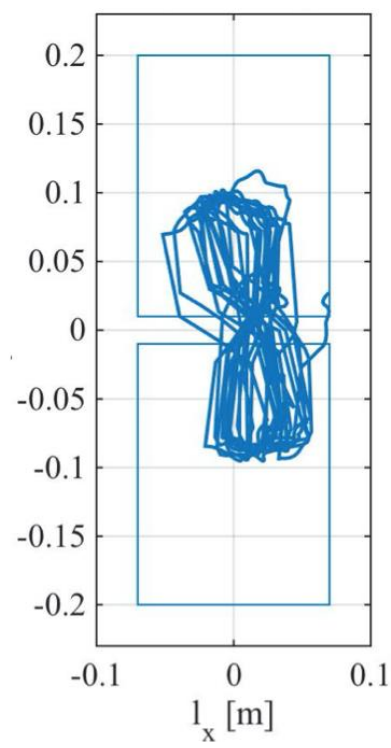

(b)

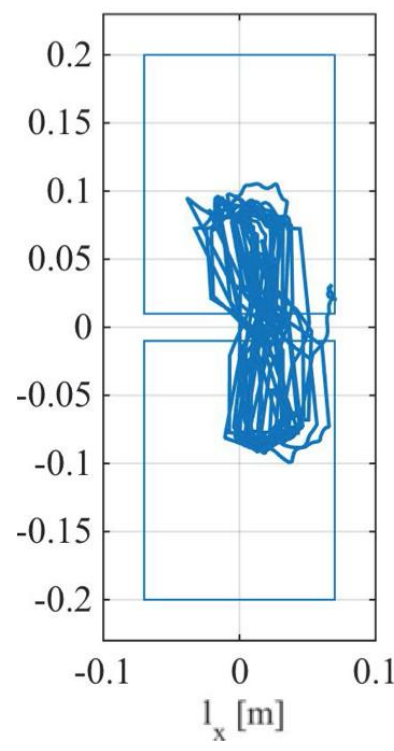

(c)

Figure 6. ZMP Lissajous at various frequencies - (a) $1.8 \pi \mathrm{rad} / \mathrm{s}$; (b) $2.1 \pi \mathrm{rad} / \mathrm{s}$; (c) $2.4 \pi$ $\mathrm{rad} / \mathrm{s}$. 


\section{CONCLUSIONS}

A 3-D active dynamic walker with telescopic knee joints based on a 3-D passive dynamic walker was developed using flat feet and ankle springs. The knee joint was composed of a telescopic actuator and oscillated by a sinusoidal function. A stable biped locomotion was excited by sinusoidal oscillation on a horizontal surface and the walking angle was varied with respect to the frequency and amplitude of the knee oscillation. The frequency response property of the locomotion was analyzed, which showed that the biped locomotion had a resonance, i.e., the frequency matched the natural frequency of locomotion in the gain property. The natural frequency indicates the most efficient frequency of the bipedal gait, because the gain property corresponds to the energy efficiency. Moreover, an " 8 " shaped ZMP trajectory was observed, which was similar to that of the human gait. The amplitude of the ZMP trajectory in the lateral direction was smaller than that in the case of RW03. This meant that RW04 did not require a large lateral oscillation. However, the simple sinusoidal oscillation, i.e., the CPG method without sensory feedback, had limitations such as stride reduction or discontinuation by phase difference. Therefore, in future work, more adaptable control strategy such as a sensory feedback using ZMP should be provided.

\section{ACKNOWLEDGEMENTS}

The authors are grateful to the Ministry of Education, Culture, Sports, Science and Technology of Japan for providing financial assistance under KAKENHI (No. 26420215).

\section{REFERENCES}

[1] McGeer T. Passive dynamic walking. Technical Report CSS-IS TR 88-02. 1988:51.

[2] Fallis GT. Walking toy. U.S. Patent no.376588; 1888.

[3] Wilson JE. Walking toy. U.S. Patent no.2140275; 1938.

[4] Coleman MJ, Ruina A. An uncontrolled walking toy that cannot stand still. Physical Review Letters. 1998;80:3658 - 61.

[5] Kuo AD. Stabilization of lateral motion in passive dynamic walking. The International journal of robotics research. 1999;18:917-30.

[6] Collins SH, Wisse M, Ruina A. A three-dimensional passive-dynamic walking robot with two legs and knees. The International Journal of Robotics Research. 2001;20:607-15.

[7] Narukawa T, Yokoyama K, Takahashi M, Yoshida K. Design and construction of a simple 3D straight-legged passive walker with flat feet and ankle springs. Journal of System Design and Dynamics. 2009;3:1-12.

[8] McGeer T. Stability and control of two-dimensional biped walking. Technical report CSS-IS TR 88-02. 1988.

[9] Collins S, Ruina A, Tedrake R, Wisse M. Efficient bipedal robots based on passive-dynamic walkers. Science. 2005;307:1082-5.

[10] Wisse M. Three additions to passive dynamic walking: actuation, an upper body, and 3D stability. International Journal of Humanoid Robotics. 2005;2:459-78.

[11] Wisse M, Van der Linde RQ. Delft pneumatic bipeds: Springer Science \& Business Media; 2007. 
[12] Narioka K, Hosoda K. Designing synergistic walking of a whole-body humanoid driven by pneumatic artificial muscles: An empirical study. Advanced Robotics. 2008;22:1107-23.

[13] Hosoda K, Takuma T, Nakamoto A, Hayashi S. Biped robot design powered by antagonistic pneumatic actuators for multi-modal locomotion. Robotics and Autonomous Systems. 2008;56:46-53.

[14] Bhounsule PA, Cortell J, Grewal A, Hendriksen B, Karssen JD, Paul C, et al. Lowbandwidth reflex-based control for lower power walking: $65 \mathrm{~km}$ on a single battery charge. The International Journal of Robotics Research. 2014;33:1305-21.

[15] Hobbelen DG, Wisse M. Ankle joints and flat feet in dynamic walking. Climbing and Walking Robots: Springer; 2005. p. 787-800.

[16] Wisse M, Hobbelen DG, Rotteveel RJ, Anderson SO, Zeglin GJ. Ankle springs instead of arc-shaped feet for passive dynamic walkers. 6th IEEE-RAS International Conference on Humanoid Robots. 2006, p. 110-6.

[17] Kinugasa T, Yoshida K, Kotake K, Fujimura K, Tanaka H, Ogawa K. 3D passive walker with ankle springs and flat feet. Journal of Robotics Society of Japan. 2009;27:91-4.

[18] Kinugasa T, Yoshida K. 3D Passive Dynamic Walkers with Flat Feet and Ankle Springs: Experiment and Analysis For Longer and More Stable Step. Proceedings of International Symposium on Mobiligence. 2009:425-30.

[19] Kinugasa T, Akiyama T, Idris MA, Yoshida K, Iribe M. Experimental analysis of 3D passive dynamic walking: Body's shape, CoM and stability. Proceedings of SICE Annual Conference. 2010, p. 1825-30.

[20] Ono K, Takahashi R, Shimada T. Self-excited walking of a biped mechanism. The International Journal of Robotics Research. 2001;20:953-66.

[21] Minakata H, Tadakuma S. An experimental study of passive dynamic walking with non-rotate knee joint biped. Proceedings of the ICASE/SICE WorkshopIntelligent Control and Systems. 2002, p. 298-303.

[22] Asano F, Luo Z. Parametrically excited dynamic walking control of telescopic legged robots. Journal Robotics Society of Japan. 2005;23:144.

[23] Miyakoshi S, Cheng G. Examining human walking characteristics with a telescopic compass-like biped walker model. IEEE International Conference on Systems, Man and Cybernetics. 2004, p. 1538-43.

[24] Kinugasa T, Osuka K, Miwa S. Biped walking by variations of knee lengths and attitude control of a body and its frequency Analysis. Journal Robotics Society of Japan. 2007;25:116.

[25] Kinugasa T, Miwa S, Yoshida K. Frequency analysis for biped walking via leg length variation. Journal of Robotics and Mechatronics. 2008;20:98.

[26] Iribe M, Furukawa H, Kojima S. Development of Compass Passive Dynamic Walking Robot with Expansion and Contraction Mechanism. Proceedings of JSME Conference on Robotics and Mechatronics. 2010. p. 2A-F04.

[27] Ito T, Kinugasa T, Akagi T, Fujimoto S, Yoshida K, Iribe M. Measurement of walking gait for 3D Passive dynamic biped walker. . Proceedings of XX IMEKO World Congress. 2012.

[28] Nishiyama S, Nomura T, Sato S. Estimation of zero moment point trajectory during human gait locomotion based on a three-dimensional rigid-link model. Technical report on ME and Bio-cybernetics of The Institute of Electronics, Information and Communication Engineers. 2002:59-64. 